\title{
Anthocyanin Copigmentation - Evaluation, Mechanisms and Implications for the Colour of Red Wines
}

\author{
Maite T. Escribano-Bailón* and Celestino Santos-Buelga \\ Grupo de Investigación en Polifenoles, Unidad de Nutrición y Bromatología, Facultad de Farmacia, Universidad de Salamanca, \\ Campus Miguel de Unamuno, E 37007 Salamanca, Spain
}

\begin{abstract}
Copigmentation is the main colour-stabilizing mechanism in plants and in food products of vegetable origin. It is a spontaneous and exothermic process that consists of the stacking of an organic molecule, called copigment, on the planar polarizable moieties of the anthocyanin coloured forms. Although this phenomenon has long been described, there are some aspects that are still not well understood or controversial like the nature of the interaction pigment to copigment, the way to quantify the extent of the process, its effect on other anthocyanin properties like astringency or reactivity. In this article a review of the most significant advances achieved in the last years in the field of intramolecular and intermolecular copigmentation is presented. Also, the most recent findings regarding wine copigments and their effects on the colour of red wines are revised.
\end{abstract}

Keywords: Copigmentation, red-wine, anthocyanin, copigment, self-association.

\section{INTRODUCTION}

Anthocyanins are purple-red pigments responsible for the colour of many flowers and fruits as well as derived foods. These pigments are structurally depending on the conditions and compositions of the media where they are dissolved and suffer interactions among them and with other compounds that influence their chemical equilibria and by the way modify their colour. Anthocyanins are usually represented by the flavylium cation, but in aqueous media this form undergoes rapid proton transfer reactions, leading to blue quinonoidal bases, and hydration, generating colorless hemiketals in equilibrium with colourless or light-yellow chalcone structures. The proportion of each form is determined by the $\mathrm{pH}$ value, and the flavylium ions predominate only in very acidic solutions [1]. Weakly acidic aqueous or hydroalcoholic solutions of pure anthocyanins are slightly coloured or almost colourless depending on the $\mathrm{pH}$ value, due to the predominant presence of colourless hydrated hemiketal forms. Nevertheless, in their natural media (plant vacuoles) as well as in food products like red wines, the anthocyanins are able to express intense red, blue or violet colours even at weakly acidic pH's. This apparent contradiction can be explained by the copigmentation phenomenon, the main colour-stabilizing mechanism in plants and in food products $[2,3]$.

Copigmentation is a spontaneous and exothermic process [4,5] that consists of the stacking of an organic molecule, called copigment, on the planar polarizable moieties of the anthocyanin coloured forms (flavylium ion or quinonoid forms). The copigment is a colourless molecule or a portion of a molecule with an electron rich $\pi$-system that is able to associate with the comparatively electronpoor flavylium ion. The nature of the interactions between pigment and copigment is still not clear; $\pi-\pi$ and $\mathrm{OH}-\pi$ interactions moderated by repulsive Coulomb-type interactions have been postulated as the forces responsible for this molecular association [5], but also charge transfer from the copigment to the flavylium cation

*Address correspondence to this author at the Grupo de Investigación en Polifenoles, Unidad de Nutrición y Bromatología, Facultad de Farmacia, Universidad de Salamanca, Campus Miguel de Unamuno, E 37007 Salamanca, Spain; Tel: +34 923294537; Fax: +34 923294515; E-mail: escriban@usal.es; csb@usal.es has been envisaged [6]. As a result, the nucleophilic attack of water at position 2 of the pyrilium nucleus, which leads to the formation of colourless hemiketal and chalcone forms, is at least partially prevented [2], which consequently increases the proportion of anthocyanin chromophores (flavylium and quinonoidal forms). The final result is that the anthocyanin solutions show a more intense colour than theoretically could be expected according to the $\mathrm{pH}$ value of the media. This hyperchromic effect is usually accompanied by a shift, in the wavelength of maximum absorbance in the visible spectrum of the anthocyanin-copigment complex, to higher values in relation to that of the free anthocyanin [7-10]. This bathochromic shift probably indicates that the copigment molecule interacts with the anthocyanin in its first excited state (electronic transition in the visible range) more strongly than with its ground-state [11], and may also reflect the reduction in the polarity of the environment of the flavylium chromophore caused by its hydrophobic association with the copigment [12]. Another possible explanation should be that since the copigmentation prevents the hydration reaction, the equilibrium between the flavylium ion and the quinonoidal form should be favoured. Thus, higher amount of the quinonoidal form should lead to bluer colours.

The variations in colour hue and intensity resulting of the interaction depend on the type of anthocyanins and copigments and their concentrations. Thus copigmentation provides at the same time colour stabilization and modification [13].

A wide variety of substances can act as copigments, e.g. organic acids, amino acids, nucleotides, metals; phenolic compounds especially flavonoids, including anthocyanins themselves. The efficiency of the copigment is conditioned by its sterical arrangement (a planar structure is found for the best copigments), but also the size plays an important role in the stabilization of the flavylium ion [5].

Two different types of copigmentation mechanisms can be distinguished: intramolecular copigmentation and intermolecular copigmentation (which includes self-association). In both cases, copigmentation complexes adopt a sandwich configuration (Fig. 1). 


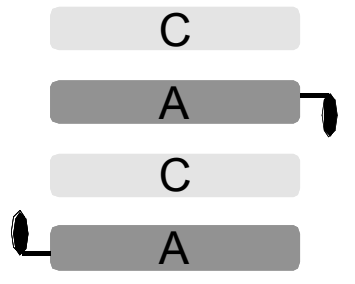

Intermolecular copigmentation

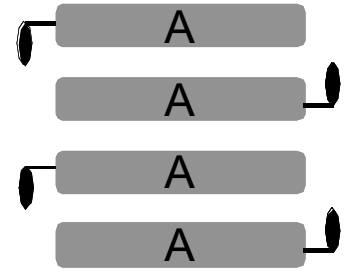

Self-association

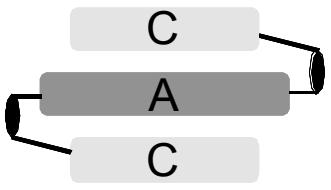

Intramolecular copigmentation

Fig. (1). Sandwich configuration of copigmentation complexes.

\section{INTERMOLECULAR COPIGMENTATION}

The intensification of the colour of anthocyanin solutions in the presence of copigments has been known since the investigations of Gertrude and Robert Robinson in the 1930's [14-17].

The intermolecular copigmentation phenomenon has been observed in both vacuolar and extra-vacuolar fluids. It is largely responsible for the diversity of flower colours that are due to a limited number of anthocyanins [18]. In media such as red wines or berry juices, copigmentation has been suggested to contribute to stabilize colour $[19,20]$ and to reduce the degradation of anthocyanins during thermal and hydrostatic high pressure processing and storage $[21,22]$. Also, it has been reported that the addition of copigments increases the half-life time of anthocyanins incorporated in yoghurt model systems [23].

In red wines, the low acidic $\mathrm{pH}$ values (3.5-4.2), together with the usual presence of sulphites, which form adducts with the anthocyanin flavylium in the same way as water nucleophilic addition to give colourless products, may lead to colour fading. Despite these facts, young red wines still show an intense and bright red colour denoting that pigment-stabilizing mechanisms exist, including copigmentation. Anthocyanin-derived pigments are formed during wine processing and storage and contribute to maintain the colour in aged red wines [24]. These newly-formed pigments might also be involved in copigmentation processes, as recently suggested in studies carried out in our laboratory [25] where a strong hyperchromic effect was observed in model solutions containing a wine pyranoanthocyanin (malvidin 3-glucoside-vinylguaiacol adduct) following addition of catechin. Indeed, pyranoanthocyanins might be expected to form even more stable copigmentation complexes than the parent anthocyanins due to their wider planar polarizable chromophore. Nevertheless, it has been also indicated that some pyranoanthocyanins do not undergo any hydration equilibrium [26]. In those cases, a hyperchromic shift resulting from a copigmentation process could not be so marked, and even not expected.

Copigmentation is an interaction only found in aqueous solutions. It is closely related to the unique associated structure of water molecules as an hydrogen-bonded tetrahedral network. This organization constitutes the most important structural parameter for copigmentation to take place. As long as it is maintained, the pigment and the copigment are forced into close contact, according to a mechanism that can be described as an hydrophobic interaction. Any factor breaking this structure (temperature increase, presence of a cosolvent, ionic strength increase ...) weakens the copigment effect $[3,27,28]$. The presence in the medium of co-solvents like ethanol, methanol, acetone or dimethylformamide provokes a partial disruption of the hydrogen-bonded network of water molecules causing a sharply decrease of the copigmentation effect. Neverthe- less, although less intense than in purer water, copigmentation still takes place in binary mixtures providing that water remains the main component of the medium [28]. Thus, it can be expected that the content of ethanol affects the extent of copigmentation in wines. In assays with de-alcoholized wines further reconstituted, Hermosín [29] found that as ethanol content increased the percentage of copigmentation diminished following a quadratic relationship, with ethanol contents between 12 and 14\% the wines maintained about three quarters of the copigmentation observed without ethanol. This observation confirms the dissociating role of ethanol on copigmentation complexes, but also shows that copigmentation still occurs in red wines. However, the bathochromic shift, that usually accompanies the hyperchromic effect in model aqueous solutions, was hardly observed in the reconstituted wines. For instance, only a weak bathochromic shift was observed in wines with ethanol content above $20-22 \%$ [29], suggesting that only some contribution of copigmentation to the blue hues of red wines containing higher ethanol levels (e.g., fortified red wines) could be expected. Kunsági-Máté et al. [30] investigated copigmentation between malvidin 3-O-glucoside and five polyphenols in model solutions and observed that both the size and the stability of the complexes of the anthocyanin with caffeic, ellagic acid, and rutin copigments increased in the presence of higher ethanol concentration. However, although the stability of the anthocyanin-procyanidin and anthocyanin-epicatechin complexes increased with increasing ethanol content, the size of the complexes was drastically reduced when the ethanol content of the solutions exceeded the critical margin of 8 vol.\%. Nevertheless, it is worth to note that these authors did not take into account the solvatochromic effect produced when changing the purely aqueous medium into one containing more or less ethanol. The authors concluded that the colour of red wine will be much more stabilized when the alcohol concentration during fermentation exceeds 8 vol.\%, although they leave a question open: do the smaller complexes between anthocyanin and flavanols above the 8 vol.\% margin significantly contribute to red wine colour?

Copigmentation may not only affect colour definition of red wines but also influence their stability. Chemical reactions that take place in wines, like oxidation and polymerization are likely to be related with the free concentrations of phenolic substrates, since a part of them is involved in the copigmentation slower reaction rates and evolution might be expected in wines with stronger copigmentation [19]. On the other hand, some authors have suggested that copigmentation could act as a first stage in the formation of new pigments and determine both the type of compounds formed and their amounts, thereby affecting the colour of aged red wines $[13,31]$. Likewise, copigmentation could also influence the extraction of pigments from the grape and their conservation in the must during winemaking [19]. 


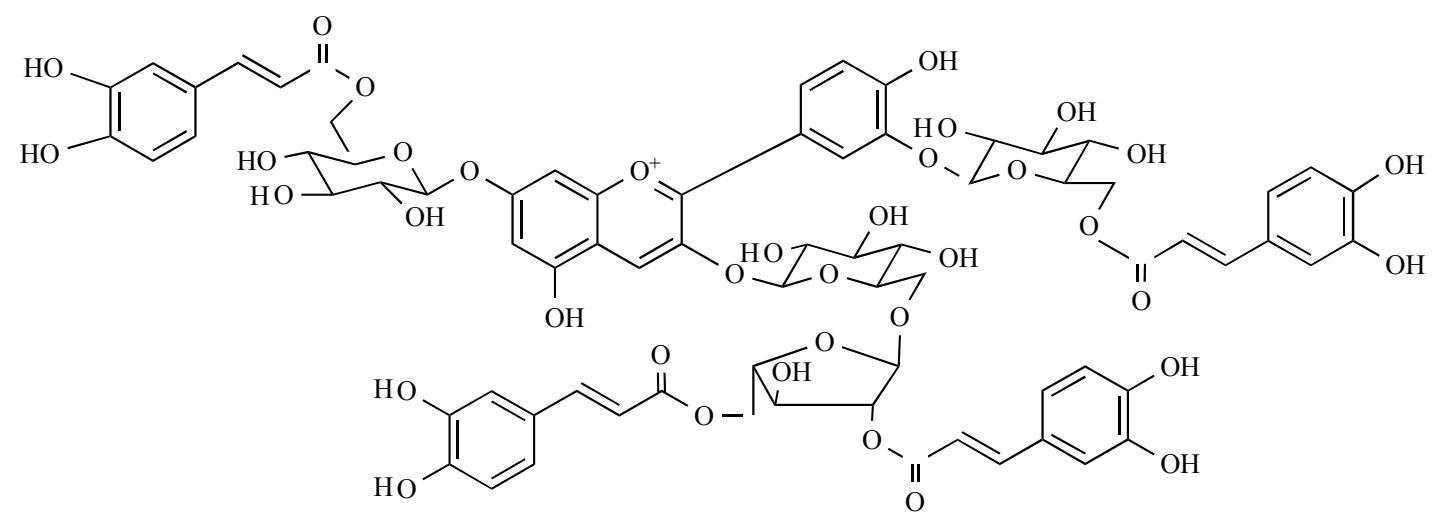

Fig. (2). Structure of zebrinin (adapted from Idaka et al., 1987 [46]).

\section{SELF-ASSOCIATION. A PARTICULAR CASE OF INTERMOLECULAR COPIGMENTATION}

Self-association consists of a positive deviation from Beer's law that occurs on increasing the concentration of anthocyanins in a given medium. The existence of this type of anthocyanin association was proved using circular dichroism and $1 \mathrm{H}$ NMR spectroscopy [32-35]. It was also shown that anthocyanin molecules stack vertically in chiral (helical) aggregates, mostly with a left-handed geometry. The formation of hydrogen bonds between the anthocyanidin-attached sugars contributes to reinforce the structure [36].

Although it is accepted that self-association contributes to the colour expression in flowers [37,38] its participation in the colour of red wines is matter of discussion. Self-association at acidic $\mathrm{pH}$ values was also found to occur between the flavylium cation itself and its $Z$-chalcone [39]. The interaction with $Z$-chalcone forms can not be expected to explain self-association in red wines, given the too low concentrations of these forms in wine. However, it might be possible the interaction between flavylium forms of different anthocyanins that could act as cofactors for each other. Somers and Evans [40] considered that self-association could be responsible for much of the non-Beer's law behaviour of red wines, whereas Boulton [19], based on studies carried out using circular dichroism, concluded that self-association was not much relevant to the enhancement of colour in young red wines, but intermolecular copigmentation between anthocyanins and different phenolic compounds would be mainly responsible for the nonlinear colour deviations observed. Assays carried out in our laboratory with anthocyanins in the concentration range $50-600 \mathrm{mg} / \mathrm{L}$, permitted us to conclude that self-association could be responsible for $8 \%$ up to $60 \%$ of the absorbance increase at $520 \mathrm{~nm}$ observed in wine-like solutions, depending on the type and concentration of the anthocyanins involved [41]. Support to the existence of self-association in wines was also provided by the observations by di Stefano et al. [42] that found that young red wines deprived of the classical copigments continued to deviate from Beer's law. This situation should change in the presence of copigments. In model assays carried out with wine anthocyanins in the presence of different mixtures of flavanols, it was observed that self-association still contribute in some extent to the increase of the solution absorbance [43]. Nevertheless, it is necessary to take into account that flavanols are poor anthocyanin copigments and that in wines more efficient copigments also exist, such as flavonols or hydroxycinnamic acids, which could favour intermolecular copigmentation with regard to self-association. Further studies are necessary to assess the actual influence of selfassociation on the colour of red wines.

\section{INTRAMOLECULAR COPIGMENTATION}

Intramolecular copigmentation may take place when the copigment is part of the anthocyanin molecule. It appears in the case of anthocyanins whose sugar residues are acylated by phenolic acids. This type of copigmentation, different from the known intermolecular copigmentation [14-17], was described by Brouillard [44,45] when investigating zebrinin (Fig. 2), an anthocyanin isolated from the flowers of Zebrina pendula that contains four aromatic acyl groups [46]. He observed that lowering of the medium free acidity did not provoke the formation of colourless species and he concluded that it was due to the aglycone (cyanidin) being selfprotected against hydration by, at least, two of its aromatic acyl residues. According to Brouillard [45], this kind of copigmentation, in which the copigment and pigment moieties are covalently bound, is more efficient than intermolecular copigmentation.

Complex acylated anthocyanins have been found to occur widely in flowers of higher plants being responsible for their exceptional colour stability [47-50]. Some edible plant materials such as black carrots with stable pigments are also known to contain high proportions of acylated anthocyanins [51].

For intramolecular copigmentation to occur, phenolic acids should be linked to the anthocyanidin through a suitable spacer, so that it allows the molecule to fold in such a way that the aromatic acyl group(s) can interact with the $\pi$-system of the planar pyrylium ring protecting it against the nucleophilic water attack [52]. This has been demonstrated by NMR spectroscopy showing the existence of long range NOEs between the aromatic acyl protons and those of the chromophore [53,54]. Also, ${ }^{1} \mathrm{H}$ NMR chemical shifts toward higher field evidence the existence of face-to-face stacking $[55,56]$. In the case of polyacylated anthocyanins, the aromatic acyl groups could simultaneously stack on both sides of the chromophore (sandwich-type complex), thereby offering high protection against hydration [12,45,57].

The high stability of the acylated anthocyanins, in the $\mathrm{pH}$ range of flavylium ions predominance, is remarkable $[58,59]$. It has been interpreted as a preference of interaction with the flavylium ion than with the quinonoidal forms [60].

Contribution of the acyl groups to the colour and stability of the anthocyanins depends on different structural factors, among which is their substitution position. Acyl residues in the B-ring stack closer than those in the A-ring [61,62], making the copigmentation process more effective. Also, large glycosyl spacers between the aglycone and the acyl moiety favour the conformational folding allowing a more efficient contact between the flavylium nucleus 
and the phenolic acid residue [12]. When the acyl group is a coumaric acid, the configuration cis or trans leads to substantial variations in the colour properties of the anthocyanin. The more coplanar arrangement of the cis isomer to the anthocyanidin moiety makes the molecule less susceptible to hydration [63].

For monoacylated anthocyanins, intermolecular copigmentation (including self-association) may also coexists with intramolecular copigmentation, since the flavylium nucleus has still one face available for stacking interactions with copigments [60], although the intermolecular contact could be hindered by the presence of large glycosyl-acyl units [12].

There is a growing interest in the search for suitable sources of stable (poly)acylated anthocyanins so that they can be used as natural colourants for food or pharmaceutical purposes.

Intramolecular copigmentation is not only attributable to complex acylated anthocyanins. Some anthocyanin derived pigments formed during maturation and ageing of red wines could also form intramolecular copigmentation complexes, as recently postulated by He et al. [64] for oligomeric pyranoanthocyanin-flavanol pigments, which would be responsible for their high stability and molar extinction coefficient. Also, Chassaing et al. [65] suggested the formation of intramolecular $\pi$-stacking in the ellagitannin-containing pigment 1 -deoxyvescalagin- $(1 ß \rightarrow 8)$-oenin.

\section{ESTIMATION OF THE COPIGMENTATION EFFECT EXTENT}

Boulton [66] proposed a method for the estimation of the copigmentation effect in red wines based on the difference of absorbance at $520 \mathrm{~nm}$ before (A) and after being diluted and later multiplied by the dilution factor (Ad), and expressed as the ratio [(A-Ad)/Ad] x 100. The dilution "breaks" all the anthocyanin complexes, either intermolecular or among anthocyanins themselves, and, therefore, the relation [(A-Ad)/Ad] x100 jointly evaluates the effect of copigmentation and self-association processes. Comparison of the absorbance before $\left(\mathrm{A}_{0}\right)$ and after addition of copigment (A) has been used to evaluate the extent of the copigmentation effect in model solutions [67], although in that case the ratio $\left[\left(\mathrm{A}-\mathrm{A}_{0}\right) / \mathrm{A}_{0}\right] \times 100$ would only estimate intermolecular copigmentation [43].

By using Boulton's method diverse authors [68-70] have concluded that copigmentation could account for up to half of the observed colour of young red wines, depending on the levels of colourless phenolic compounds or cofactors present (e.g. ethanol levels, acetaldehyde, metals...), which could even have more importance than anthocyanins content to define the colour in those wines.

During wine life a decrease is produced in the concentrations of both anthocyanins and copigments in the wines, so that the extent of copigmentation also decreases until having no influence on the colour of aged red wines. Results obtained about the contribution of copigmentation to red wine colour differ from one author to another. Hermosin et al. [71], in assays with Spanish wines of the varieties Cencibel (synonym of Tempranillo), Cabernet-Sauvignon and Syrah, found that copigmentation accounted for $32-45 \%$ of the colour of red wines recently elaborated to decrease to $20-34 \%$ after 3 months and be practically negligible after 9 months (0-5\%). In studies carried out with wines made from grape varieties usually growing on the Canary Islands (Listan Negro and Negramoll), Darias-Martin et al. [68] calculated that copigmentation contributed to $22 \%$ and $19 \%$ of colour in red wines of 1 and 2 years of age, respectively. Similar results were obtained by Lorenzo et al. [72] who estimated that around $18 \%$ of copigmentation still exists in their wines after 9 months of oak ageing. A contribution ranging from 8 to $30 \%$ was obtained by Vesari et al. [73] in the case of young red wines. The discrepancy between the results might be explained by variations of wine composition, as determined by grape characteristics and winemaking processes, which provoke differences in the type and levels of anthocyanins and cofactors that affect the extent and stability of the copigmentation process. Further, the conditions of storage and ageing, as well as scale of vinifications should also play a determinant role on the results obtained. On the other hand, as indicated above, presence of newly formed anthocyanin-derived pigments in red wines, in addition to their direct contribution to the colour, might also account for the extent of copigmentation, as some of them have been suggested to be able to participate in such processes $[25,64]$.

Boulton's method only takes into account the variation at $\lambda_{\max }$ of the flavylium ion in the visible region, thus ruling out the modifications that copigmentation induces in other regions of the visible spectrum that have qualitative and quantitative influence on the colour. Gonnet [74] proposed another approach based on the colourimetric analysis in the CIELAB colour space that considers the spectral changes over the complete range of visible wavelengths. Some authors have employed this methodology to evaluate the changes in lightness, chromaticity and hue that the copigmentation process induces in the colour so as to quantify the colour difference that this phenomenon implies [67,74-76]. The hyperchromic effect produced by copigmentation is connected with a darkening of the solutions and with a greater quantity of colour and visual saturation. The colour differences $\left(\Delta \mathrm{E}^{*} \mathrm{ab}\right)$ between the copigmented and non-copigmented solutions can be calculated as the Euclidean distance between two points in the three-dimensional space defined by $\mathrm{L}^{*}, \mathrm{a}^{*}$ and $\mathrm{b}^{*}: \Delta \mathrm{E}^{*} \mathrm{ab}=\left[\left(\Delta \mathrm{L}^{*}\right)^{2}+\left(\Delta \mathrm{a}^{*}\right)^{2}+\left(\Delta \mathrm{b}^{*}\right)^{2}\right]^{1 / 2}$. It has been indicated that the human eye is able to discriminate two colours when $\Delta \mathrm{E}^{*} \mathrm{ab} \geq 1[74,77]$, but when the wine is observed through standardized wine tasting glasses, the colour discrimination is worse and the acceptable tolerance for the human eye in distinguishing the colour of wines might be around 3.0 CIELAB units [78].

\section{WINE COPIGMENTS}

Different types of compounds have been evaluated as potential anthocyanin copigments, including alkaloids, amino acids, nucleotides, carbohydrates and phenolic compounds [7,28,79]. Many of these compounds are to be found in wine, although some of them should not be expected to have a relevant contribution to the copigmentation phenomenon in red wine, due to low levels or comparatively poor ability to act as anthocyanin copigments. Thus, sugars (i.e., fructose and glucose) have been found to produce no change or a slight increase in the absorbance of anthocyanin solutions, even at very high concentrations ( $50 \%$ in water) [80]. Similar observations were made for polysaccharides like pectins [80] or mannoproteins. Guadalupe et al. [81], observed that wines elaborated with addition of commercial mannoproteins or inoculation of must with yeast over-expressing mannoproteins, had lower values of colour intensity than controls at the end of fermentation and that the differences increased during malolactic fermentation and ageing; such an observation would question the usual belief that polysaccharides may protect wine colour.

Benzoic acids (gallic acid, protocatechuic acid, etc.), which can be transferred from the grape, released from barrels wood or de- 


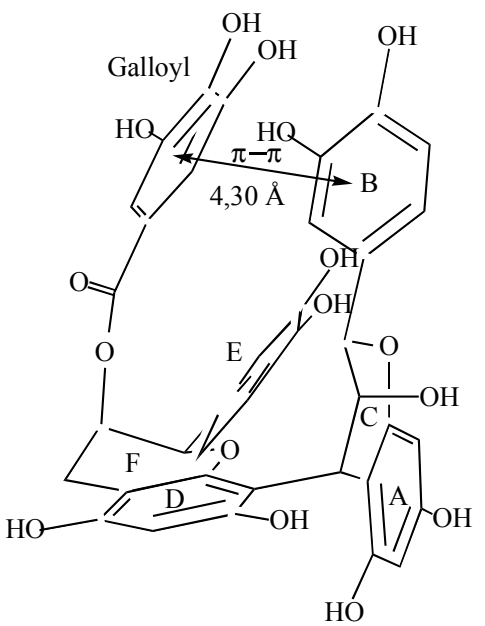

Dimer B2-3"-O-gallate (B2G)

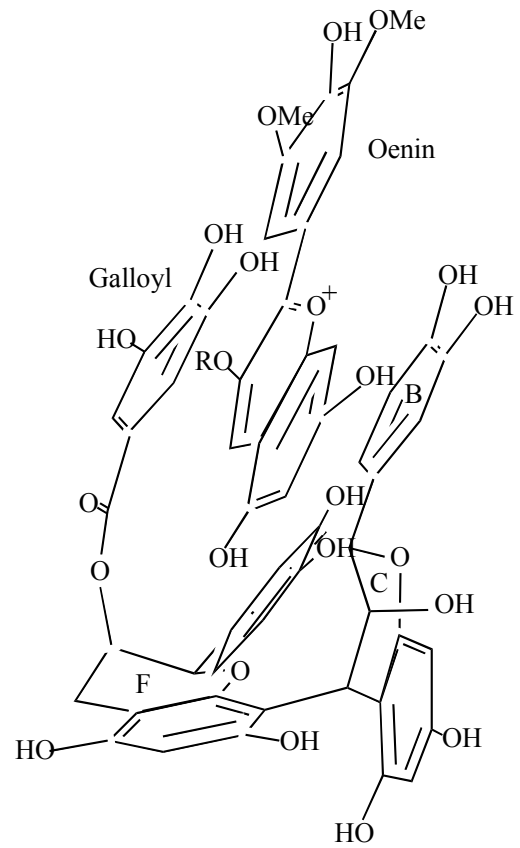

B2G/oenin sandwich-type complex

Fig. (3). Preferred conformation of dimer B2-3'-O-gallate (B2G) and suggested conformational arrangements of oenin intercalated between the galloyl ester group and catechol ring B of B2G (adapted from Berke and De Freitas 2005 [97]).

rived from anthocyanin breakdown, are considered poor anthocyanin copigments compared with other phenolic compounds $[28,82]$. By contrast, some hydrolysable tannins could be potentially good copigments [79], but their presence in wine is restricted to wood-aged wines, in which the concentration of anthocyanins is already reduced. Furthermore, although notable variations may exist depending on the type and age of the barrel, the contents of this type of tannins in wine are usually very low. Thus, concentrations of a few $\mathrm{mg} / \mathrm{L}$ were found for ellagic acid and ellagitannins after ageing in oak barrels of different origins [83,84], French oak and new barrels releasing more ellagitannins than American oak [83].

Among red wine components, flavonoids (in particular, flavonols and flavanols) and hydroxycinnamoyl derivatives appear as the more promising compounds featuring anthocyanin copigments. In assays carried out using model solutions, flavonols were demonstrated to behave as powerful anthocyanin copigments [4,7,85-87], whereas flavan-3-ols are comparatively poor cofactors with hydroxycinnamic acids showing intermediate behaviors [28,67]. However, studies dealing with red wines are scarce, so that the actual influence of all these compounds on colour is rather speculative.

Amounts of the different compounds able to participate in copigmentation are extremely variable in red wines, depending on the characteristics of grapes and winemaking processes. In general, it is assumed that contents of flavonols and hydroxycinnamoyl derivatives are much lower than those of flavanols, which, as either monomers (catechins) or polymers (i.e., condensed tannins, proanthocyanidins), constitute the majority of the phenolic components in red wines [88]. Flavonols are found in grapes as glycosides (mainly in the form of quercetin 3-glucuronide, quercetin 3-glucoside and myricetin 3-glucoside), which are transferred to wine during maceration. In the course of wine life certain amounts of the aglycones are released as a result of the cleavage of the glycosidic linkages. Contents of total flavonols in young red wines range from traces to values around $100 \mathrm{mg} / \mathrm{L}$, depending on the grape variety and winemaking procedures $[71,89,90]$, with a decrease in their levels after 9 months between $50 \%$ and $80 \%$ of the original contents [71]. Hydroxycinnamic acids (caffeic, $p$-coumaric, and ferulic acids) appear in wine as a consequence of the hydrolysis of grape hydroxycinnamoyltartaric esters (i.e, caftaric, coutaric and fertaric acids). Further they can also be released from the barrel wood and minor amounts are also produced by cleavage of the acylated anthocyanins. Thus, contrary to flavonols, their levels could increase during red wine life. Concentrations of total hydroxycinnamic derivatives in red wines seem to be in the same order of magnitude as flavonols (some tens of $\mathrm{mg} / \mathrm{L}$ ) [88,91].

Flavanols are the more abundant phenolic compounds in red wines, with contents that may reach some hundreds $\mathrm{mg} / \mathrm{L}$ [92] or be even in the level of $\mathrm{g} / \mathrm{L}$ when taking into account the badly characterized and quantified proanthocyanidin polymers [88]. Thus, in spite of being in general worse copigments than flavonols or hydroxycinnamates, it is assumed that flavanols should play a role in relation to red wine colour. Indeed, the importance of tannins to provide support to colour and ensure its stabilization during ageing in red wines is a fact empirically assumed in enology.

The comparatively smaller ability of flavanols to act as copigments is attributed to their non-planar structure that impedes a closer approach to the anthocyanin and reduces the potential surface area available for hydrophobic stacking. However, important differences can exist among flavanols regarding their efficiency as anthocyanin copigments. Thus, regarding flavanol monomers, epicatechin is a better copigment than catechin $[28,93,94]$, which is explained by the preferential quasi-equatorial conformation of its ring B that allows both aromatic nuclei to be approximately coplanar and participate in the $\pi-\pi$ stacking with the flavylium or quinonoidal forms of the anthocyanins [93]. On the other hand, procyanidin dimers with C4-C6 interflavanic linkages seem to be better copigments than their respective $\mathrm{C} 4-\mathrm{C} 8$ dimers [95], possibly due to the fact that they possess a more flexible and open conformational 


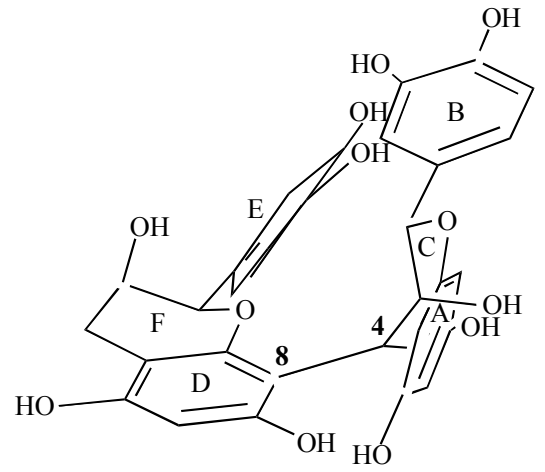

Dimer B2 (C4-C8)

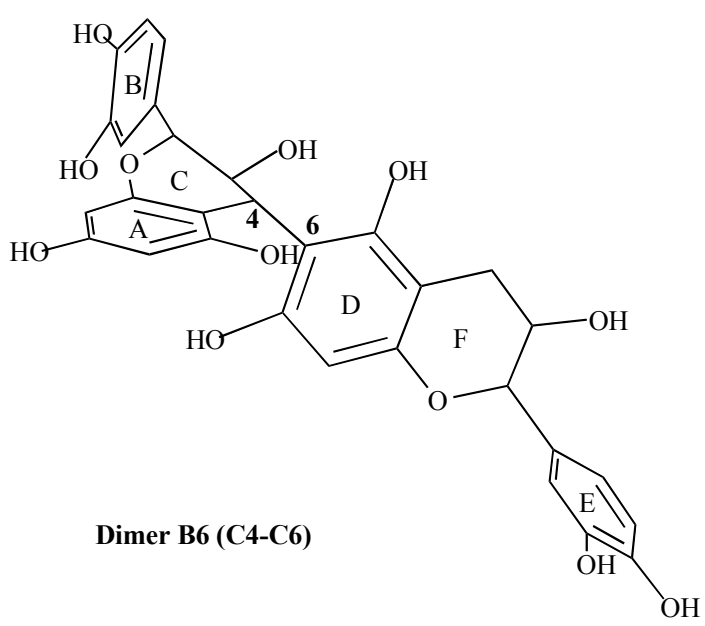

Fig. (4). Representations of the preferred conformations of procyanidin dimers B2 (epicatechin-4,8-epicatechin) and B6 (epicatechin-4,6-epicatechin) (adapted from De Freitas et al. 1998 [96]).

structure that allows them better interaction with the anthocyanin (Fig. 4) [96]. Moreover, galloylation at C-3 of the catechin units improves the ability of flavan-3-ols to act as copigmente [93,97]. The substantial enhancement of copigmentation in the case of the B2-3'-O-gallate dimer compared to its analogue dimer B2 could be explained by the presence of the well exposed planar $\pi-\pi$ systems forming a pocket into which the anthocyanin may intercalate, thereby offering an important interaction (Fig. 3) [97].

Studies about flavanols copigmentation ability have mostly been carried out with monomers and dimers, and little is known about efficiency of compounds with larger degree of polymerisation. Some authors $[67,95,98]$ found procyanidin dimers B3 and B2 to be worse anthocyanin copigments than their constituting monomers (catechin and epicatechin), which was explained by the existence of conformational restraints imposed by the increase of the molecular size, whereas Malien-Aubert et al. [99] did not observe great differences among flavanols up to the tetramer regarding their efficiency as copigments. However, these authors observed that in the course of time procyanidin trimers and tetramers protected the red colour in the solutions more efficiently than do monomers and dimers, which induced the formation of xanthylium pigments leading to a yellowing of the solutions. The ability of the flavanols to maintain the colour of the anthocyanin solutions was also determined by their stabilities. Thus, the procyanidin B3 was more susceptible to thermal degradation than B2 and produced a faster alteration in the colour [99]. More recently, in studies carried out with condensed tannin analogues consisting of ethyl-bridged oligomers containing up to 6 catechins units obtained by acetaldehydemediated condensation, it was found that dimers and trimers were more effective as malvidin 3-glucoside copigments than monomers and compounds with higher degree of polymerisation [100]. All in all, more studies are required to conclude about the influence of the polymerisation degree of flavanols on the copigmentation effect.

The greater or lesser ability of pure compounds to act as anthocyanin copigments does not ensure an adequate maintenance of the colour. In assays carried out in model solutions, it was observed that although flavonols are good copigments, they might not provide a suitable preservation of the colour with the passage of time. Flavonols induce a pure copigmentation effect, which decreases as their concentration in the medium declines, being apparently re- placed by other cofactors, such as hydroxycinnamic acids and flavanols, which are able to react with the anthocyanins to yield new pigments that help the colour to be kept $[67,71]$.

Pre-fermentation addition of different copigments during winemaking has been tried by some authors as a strategy to improve wine colour. For instance, Darias-Martín et al. [101] in experimental red wines made with autochthonous grapes of the $\mathrm{Ca}$ nary Islands (i.e., 'Listan Negro' and 'Negramoll') observed enhancements of the colour (measured as the increase in the absorbance at $520 \mathrm{~nm}$ ) of $13 \%$ and $60 \%$ in the wines added with catechin and caffeic acid $(120 \mathrm{mg} / \mathrm{L}$ each), respectively, in relation to the controls. However, the wines added with catechin lost their red colour more rapidly than the control wines, whereas those added with caffeic acid still showed $23 \%$ more colour than the controls after 210 days of storage. In a further assay, the same group found that the enhancement in the colour was greater when the amount of caffeic acid added increased (in the range 120-960 mg/L), reaching up to $111 \%$ of increase in the absorbance at $520 \mathrm{~nm}$; this effect was more noticeable in wines made with cultivars less rich in colour [102].

Bloomfield et al. [103] in wines made adding caffeic and $p$ coumaric acids to 'Cabernet Sauvignon' and 'Pinot noir' grapes after crushing, also found an enhancement of the colour due to copigmentation, $p$-coumaric acid being more effective than caffeic acid. Nevertheless, the fact that these authors observed a hypsochromic shift in the wavelength of maximum absorption in the visible region of the wines spectra pointed out that not only a copigmentation effect was produced, but also that the presence of newlyformed pyranthocyanins pigments should be taken into account.

In wines made with pre-fermentative addition of rutin, caffeic acid or $p$-coumaric acid to 'Cabernet Sauvignon' and 'Tempranillo' grapes, Schwarz et al. [70] observed that only the addition of rutin was always accompanied by a significant hyperchromic shift at 520 nm ( $9 \%$ for 'Tempranillo' and 35\% for 'Cabernet Sauvignon' wines) explainable by a copigmentation effect. However, the addition of hydroxycinnamic acids had variable effects depending on structure and grape cultivar. A hyperchromic effect was only found when $p$-coumaric acid was added to 'Cabernet Sauvignon' wine ( $22 \%$ increase of $A 520$ ), whereas the pre-fermentation addition of caffeic acid always resulted in hypochromic effects, contrary to the results previously obtained by other authors. In further analyses 
carried out after 4 months of storage the percentage of colour due to copigmentation decreased in all the wines, and after 9 months no colour attributable to copigmentation was noticeable.

Assays were also made with addition of grape seeds (supplementary amounts of $60 \mathrm{~g} / \mathrm{L}$ ) during fermentation, so as to provide supplementary sources for the extraction of proanthocyanidins in 'Garnacha', 'Tempranillo' and 'Vranac' red wines [104,105]. In all cases, wines obtained showed greater contents of total phenolics and free anthocyanins, as well as an increase in their colour intensity and a stabilization of wine colour through time. However, these benefits were not produced when higher amounts of seeds were added (120 g/L), which was explained by an increased retention of the colouring matter by pomace. The enrichment in seeds during wine making was also seen to lead to wines with a greater concentration of total anthocyanins in red wines obtained by microvinification by Canals et al. [106], although that increase was slight and not statistically significant. Nevertheless, the amounts of seeds added by these authors (up to $384 \mathrm{~g}$ added to $12 \mathrm{~kg}$ of grapes) were lower than those used in the studies of Kovac and coworkers [104,105]. Similar results were also obtained in wines subjected to delestage, a practice to strengthen maceration [106].

An interesting observation made by all these authors was that the initial increase in the colour of the wines made with prefermentative addition of cofactors of either nature (i.e., flavonols, hydroxycinnamic acids or flavanols) was not only explained by a copigmentation effect but also by an enhanced anthocyanin extraction from grapes. This seems to support the views of Boulton [19] that colour extraction and retention in the wine is not only depending on the concentrations of pigments in the berry, but also on the levels of cofactors. Hence the final contents of anthocyanins in the wine would be fixed once saturation has been reached for the major cofactors, the extent of this equilibrium being predetermined by the composition of the grape. Thus, according to this author the factors controlling the solubility and retention of pigments in young wines would be more important than contacting methods in determining wine colour. The incorporation of extra copigments, either by external addition or co-vinification of different grape varieties contributing additional cofactors, would shift the extraction equilibrium out causing more pigment to move from the skins into the wine. Further, the increase in the anthocyanin concentration in the must would induce more copigments to be extracted from the grapes, thus the levels of other phenolics in the wines would also be increased. Experimental support to this view has been contributed by Lorenzo et al. [72] in studies where 'Monastrell' grapes were cofermentated in the presence of 'Cabernet Sauvignon' and 'Merlot' grapes.

The increased phenolic extraction provided by copigmentation should not only have implications on the colour but also on other sensory properties, as well as on the redox characteristics of the wines, as many of these compounds show recognized antioxidant and oxygen scavenging capacities. It has been observed that the complex formed from malvin and hydroxycinnamic acids showed better antioxidative properties than pure malvin, but weaker than the pure acids [107].

Also, it is known that flavanols display different degrees of astringency and bitterness. According to Boulton [19], their rates of binding to saliva proteins and receptors in the mouth could be related to their free concentrations rather than to their total concentrations. Therefore, although the anthocyanins themselves may not contribute to taste, they could somehow modulate astringency and bitterness in wines perception through the retention of flavanols and other phenolics in the copigmentation complexes thus reducing the levels of their free forms. Alvarez et al. [108] found that prefermentative addition of flavanol-derived copigments produces wines with a lower astringency index, calculated as capacity of precipitation of ovalbumin, which was explained by astringent components included in the copigmentation stacks and not free for binding proteins.

\section{ACKNOWLEDGEMENTS}

Thanks are due to the Spanish MICINN (Project ref. AGL200805569-C02-01 and Consolider-Ingenio 2010 Programme ref. FUNC-FOOD, CSD2007-00063) and to Junta de Castilla y León (group GR133) for financial support.

\section{REFERENCES}

[1] Brouillard, R.; Delaporte, B. Chemistry of Anthocyanin Pigments. 2. Kinetic and Thermodynamic Study of Proton Transfer, Hydration, and Tautomeric Reactions of Malvidin 3-Glucoside. J. Am. Chem. Soc., 1977, 99(26), 84618468 .

[2] Mazza, G.; Brouillard, R. Recent Developments in the Stabilization of Anthocyanins in Food Products. Food Chem., 1987, 25(3), 207-225.

[3] Mazza, G.; Brouillard, R. The Mechanism of Co-Pigmentation of Anthocyanins in Aqueous Solutions. Phytochemistry, 1990, 29(4), 1097-1102.

[4] Baranac, J.M.; Petranovic, N.A.; Dimitric-Marcovic, J.M. Spectrophotometric Study of Anthocyanin Copigmentation Reactions. J. Agric. Food Chem., 1996, 44(5), 1333-1336.

[5] Kunsági-Máté, S.; Szabo, K.; Nikfardjam, M.P.; Kollar, L. Determination of the Thermodynamic Parameters of the Complex Formation Between Malvidin-3-O-Glucoside and Polyphenols. Copigmentation Effect in Red Wines. J. Biochem. Biophys. Methods, 2006, 69(1-2), 113-119.

[6] Ferreira da Silva, P.; Lima, J.C.; Freitas, A.A.; Shimizu, K.; Maçanita, A.L.; Quina, F.H. Charge-Transfer Complexation as a General Phenomenon in the Copigmentation of Anthocyanins. J. Phys. Chem. A, 2005, 109(32), 73297338.

[7] Asen, S.; Stewart, R.N.; Norris, K.H. Co-Pigmentation of Anthocyanin in Plant Tissues and its Effect on Colour. Phytochemistry, 1972, 11(3), 11391144.

[8] Chen, L.J.; Hrazdina, G. Structural Aspects of Anthocyanin-Flavonoid Complex Formation and its Role in Plant Color. Phytochemistry, 1981, 20(2), 297-303.

[9] Mazza, G.; Miniati, E. Anthocyanins in Fruits, Vegetables and Grain. Ist Edn., CRC Press: Boca Raton, FL., 1993

[10] Dangles, O. Anthocyanin Complexation and Colour Expression. Analusis Mag., 1997, 25(8), 50-52.

[11] Alluis, B.; Pérol, N.; Elhajji, H.; Dangles, O. Water-Soluble Flavonol (=3Hydroxy-2-Phenyl-4H-1-Benzopyran-4-One) Derivatives: Chemical Synthesis, Colouring, and Antioxydant Properties. Helv. Chim. Acta, 2000, 83(2), 428-443.

[12] Dangles, O.; Wigand, M.C.; Brouillard, R. Polyphenols in Plant Pigmentation: The Copigmentation Case. J. Agric. Food Chem., 1992, 41(5), 209-216.

[13] Brouillard, R.; Chassaing, S.; Fougerousse, A. Why Are Grape/Fresh Wine Anthocyanins So Simple and Why Is It that Red Wine Color Lasts So Long? Phytochemistry, 2003, 64(7), 1179-1186.

[14] Robinson, R.; Robinson, G.M. A Survey of Anthocyanins. I. Biochem. J., 1931, 25, 1687-1705.

[15] Robinson, G.M.; Robinson, R. A Survey of Anthocyanins. II. Biochem. J., 1932, 26(5), 1647-1664.

[16] Robinson, G.M.; Robinson, R. A survey of anthocyanins. III. Biochem. J., 1933, 27(1), 206-212.

[17] Robinson, G.M.; Robinson, R. A Survey of Anthocyanins. IV. Biochem. J. 1934, 28(5), 1712-1720.

[18] Brouillard, R.; Wigand, M.C.; Cheminat, A. Loss of Colour, a Prerequisite to Plant Pigmentation by Flavonoids. Phytochemistry, 1990, 29(11), 3457-3460.

[19] Boulton, R. The Copigmentation of Anthocyanins and Its Role in the Color of Red Wine: A Critical Review. Am. J. Enol. Vitic., 2001, 52(2), 67-87.

[20] Rein, M.J.; Heinonen, M. Stability and Enhancement of Berry Juice Color. $J$. Agric Food Chem., 2004, 52(10), 3106-3114.

[21] Mollov, P.; Mihalev, K.; Shikov, V.; Yoncheva, N.; Karagyozov, V. Colour Stability Improvement of Strawberry Beverage by Fortification with Poly- 
phenolic Copigments Naturally Occurring in Rose Petals. Inn. Food Sci. Emerg. Technol., 2007, 8(3), 318-321.

[22] Del Pozo-Insfran, D.; Del Follo-Martinez, A.; Talcott, S.T.; Brenes, C.H. Stability of Copigmented Anthocyanins and Ascorbic Acid in Muscadine Grape Juice Processed by High Hydrostatic Pressure. J. Food Sci., 2007, 72(4), 247-253.

[23] Bordignon-Luiz, M.T.; Gauche, C.; Gris, E.F.; Falcao, L.D. Colour Stability of Anthocyanins from Isabel Grapes (Vitis labrusca L.) in Model Systems. LWT-Food Sci. Technol., 2007, 40(4), 594-599.

[24] Santos-Buelga, C.; Freitas, V. Influence of Phenolics on Wine Organoleptic Properties. In Wine Chemistry and Biochemistry; Moreno-Arribas; Polo, Eds. Springer Science: New York, 2009; pp. 529-570.

[25] Quijada-Morín, N.; Dangles, O.; Rivas-Gonzalo, J.C.; Escribano-Bailón, M.T. Physico-Chemical and Chromatic Characterization of Malvidin 3Glucoside-Vinylcatechol and Malvidin 3-Glucoside-Vinylguaiacol Wine Pigments. J. Agric. Food Chem., 2010, 58(17), 9744-9752.

[26] Oliveira, J.; Mateus, N.; Silva, A.M.S. Freitas, V. Equilibrium Forms of Vitisin B Pigments in an Aqueous System Studied by NMR and Visible Spectroscopy. J. Phys. Chem. B, 2009, 113(32), 11352-11358.

[27] Brouillard, R.; Mazza, G.; Saad, Z.; Albrecht-Gary, A.M.; Cheminat, A. The Copigmentation Reactions of Anthocyanins: A Microprobe for the Structural Study of Aqueous Solution. J. Am. Chem. Soc., 1989, 111 (7), 2604-2610.

[28] Brouillard, R.; Wigand, M.C.; Dangles, O.; Cheminat, A. pH and Solvent on the Copigmentation Reaction on Malvin with Polyphenols, Purine and Pyrimidine Derivatives. J. Chem. Soc. Perkin Trans. 2, 1991, 1235-1241.

[29] Hermosin, I. Influence of Ethanol Content on the Extent of Copigmentation in a Cencibel Young Red Wine. J. Agric. Food Chem., 2003, 51(14), 40794083

[30] Kunsági-Máté, S; Ortmann, E; Kollár, L; Nikfardjam, M.P. Entropy-Driven Complex Formation of Malvidin-3-O-Glucoside with Common Polyphenols in Ethanol-Water Binary Solutions. Spectrochim. Acta A, 2008, 70(4), 860865 .

[31] Brouillard, R.; Dangles, O. (1993). Flavonoids and Flower Colour. In The Flavonoids. Advances in research since 1986; J.B. Harborne, Ed.; Chapman \& Hall: London, 1993; pp. 565-586.

[32] Hoshino, T.; Matsumoto, U.; Goto, T.; Harada, N. Evidence for the Selfassociation of Anthocyanins. IV. PMR Spectroscopic Evidence for the Vertical Stacking of Anthocyanin Molecules. Tetrahedron Lett., 1982, 23(4), 433436.

[33] Hoshino, T.; Goto, T. Effects of $\mathrm{pH}$ and Concentration on the Selfassociation of Malvin Quinonoidal Base-Electronic and Circular Dichroic Studies. Tetrahedron Lett., 1990, 31 (11), 1593-1596.

[34] Hoshino, T. Hoshino T. Self-Association of Flavylium Cations of Anthocyanidin 3,5-Diglucosides Studied by Circular Dichroism and ${ }^{1} \mathrm{H}$ NMR. Phytochemistry, 1992, 31(2), 647-653.

[35] Goto, T.; Kondo, T. Structure and Molecular Stacking of AnthocyaninsFlower Color Variation. Angew. Chem. Int. Ed. Engl., 1991, 30(1), 17-33.

[36] Ellestad, G.A. Structure and Chiroptical Properties of Supramolecular Flower Pigments. Chirality, 2006, 18(2), 134-144.

[37] Hoshino, T.; Matsumoto, U.; Harada, N.; Goto, T. Chiral Exciton Coupled Stacking of Anthocyanins: Interpretation of the Origin of Anomalous CD Induced by Anthocyanin Association. Tetrahedron Lett., 1981, 22 (37), pp 3621-3624.

[38] Hoshino, T. Anthocyanin self-aggregates .6. An Approximate Estimate of Self-Association Constants and the Self-Stacking Conformation of Malvin Quinonoidal Bases Studied by H-1-NMR. Phytochemistry, 1991, 30(6), 2049-2055.

[39] Houbiers, C.; Lima, J. C.; Maçanita, A.L.; Santos, H. Color Stabilization of Malvidin 3-Glucoside: Self-Aggregation of the Flavylium Cation and Copigmentation with the Z-Chalcone Form. J. Phy. Chem. B, 1998, 102(18), 35783585 .

[40] Somers, T.C.; Evans, M.E. Grape Pigment Phenomena: Interpretation of Major Colour Losses During Vinification. J. Sci. Food Agric., 1979, 30(6), 623-633.

[41] González-Manzano, S.; Santos-Buelga, C.; Dueñas, M.; Rivas-Gonzalo, J.C.; Escribano-Bailón, M.T. Colour Implications of Self-Association Processes of Wine Anthocyanins. Eur. Food Res. Tech., 2008, 226(3), 483-490.

[42] Di Stefano, R.; Gentilini, N.; Panero, L. Osservazioni Sperimentali sul Fenomeno della Copigmentazione. Riv. Vit. Enol., 2005, 58(1), 35-50.

[43] González-Manzano, S.; Dueñas, M.; Rivas-Gonzalo, J.C.; Escribano-Bailón, M.T.; Santos-Buelga, C. Studies on the Copigmentation between Anthocyanins and Flavan-3-ols and Their Influence in the Colour Expression of Red Wine. Food Chem., 2009, 114(2), 649-656.

[44] Brouillard, R. The Chemistry of Anthocyanin Pigments .7. Origin of the Exceptional Color Stability of the Zebrina Anthocyanin. Phytochemistry, 1981, 20(1), 143-145.
[45] Brouillard, R. Chemical Structure of Anthocyanins. In Anthocyanins as Food Colors. Markakis, Ed.; Academic Press Inc.: New York, 1982; pp. 1-40.

[46] Idaka, E.; Ohashi, Y.; Ogawa, T.; Kondo, T.; Goto, T. Structure of Zebrinin, a Novel Acylated Anthocyanin Isolated from Zebrina pendula. Tetrahedron Lett., 1987, 28(17), 1901-1904.

[47] Harborne, J.B.; Boardley, M. The Widespread Occurrence in Nature of Anthocyanins as Zwitterions. Z. Naturforsch., 1985, 40c(5-6), 305-308.

[48] Harborne, J. B. The Natural Distribution in Angiosperms of Anthocyanins Acylated with Aliphatic Dicarboxylic Acids. Phytochemistry, 1986, 25(8), 1887-1894.

[49] Takeda, K.; Harborne, J.B.; Self, R. Identification and Distribution of Malonated Anthocyanins in Plants of the Compositae Phytochemistry, 1986, 25(6), 1337-1342.

[50] Saito, N.; Harborne, J.B. Correlations between Anthocyanin Type, Pollinator and Flower Colour in the Labiatae. Phytochemistry, 1992, 31(9), 3009-3015.

[51] Giusti, M.M.; Wrolstad, R.E. Acylated Anthocyanins from Edible Sources and Their Applications in Food Systems. Biochem. Eng. J., 2003, 14(3), $217-$ 225.

[52] Dangles, O.; Saito, N.; Brouillard, R. Anthocyanin Intramolecular Copigment Effect. Phytochemistry, 1993, 34(1), 119-124.

[53] Gläßgen, W.E.; Wray, V.; Strack, D.; Metzger, J.W.; Seitz, H.U. Anthocyanins from Cell Suspension Cultures of Daucus carota. Phytochemistry, 1992 31(5), 1593-1601.

[54] Yoshida, K.; Kondo, T.; Goto, T. Intramolecular Stacking Conformation of Gentiodelphin, a Diacylated Anthocyanin from Gentiana makinoi. Tetrahedron, 1992, 48(21), 4313-4326.

[55] Goto, T. Structure, Stability and Color Variation of Natural Anthocyanins. Prog. Chem. Org. Nat. Prod., 1987, 52, 113-158.

[56] Goto, T.; Tamura, H.; Kawai, T.; Hoshino, T.; Harada, N.; Kondo, T. Chemistry of Metalloanthocyanins. Ann. N. Y. Acad. Sci., 1986, 471, 155-173.

[57] Rodriguez-Saona, L.E.; Giusti, M.M.; Wrolstad, R.E. Color and Pigment Stability of Red Radish and Red-Fleshed Potato Anthocyanins in Juice Model Systems. J. Food Sci., 1999, 64(3), 451-456.

[58] Shi, Z.; Francis, F.J.; Daun, H. Quantitative Comparison of the Stability of Anthocyanins from Brassica oleracea and Tradescantiaa pallida in NonSugar Drink Model and Protein Model System. J. Food Sci., 1992, 57(3), 768-770.

[59] Baublis, A.; Spomer, A.; Berber-Jiménez, M.D. Anthocyanin Pigments: Comparison of Extract Stability. J. Food Sci., 1994, 59(6), 1219-1221.

[60] Malien-Aubert, C.; Dangles, O.; Amiot, M.J. Color Stability of Commercial Anthocyanin-Based Extracts in Relation to the Phenolic Composition. Protective Effects by Intra and Intermolecular Copigmentation. J. Agric. Food Chem., 2001, 49(1), 170-176.

[61] Yoshida, K. Toyama, Y.; Kameda, K.; Kondo, T. Contribution of Each Caffeoyl Residue of the Pigment Molecule of Gentiodelphin to Blue Color Development. Phytochemistry, 2000, 54(1), 85-92.

[62] Bloor, S.J. Deep Blue Anthocyanins from Blue Dianella Berries. Phytochemistry, 2001, 58(6), 923-927.

[63] George, F.; Figueiredo, P.; Toki, K.; Tatsuzawa, F.; Saito, N.; Brouillard, R. Influence of Trans-Cis Isomerisation of Coumaric Acid Substituents on Colour Variance and Stabilisation in Anthocyanins. Phytochemistry, 2001, 57(5), 791-795.

[64] He, J.; Carvalho, A.R.F.; Mateus, N.; De Freitas, V. Spectral Features and Stability of Oligomeric Pyranoanthocyanin-Flavanol Pigments Isolated from Red Wines. J. Agric. Food Chem., 2010, 58(16), 9249-9258.

[65] Chassaing, S.; Lefeuvre, D.; Jacquet, R.; Jourdes, M.; Ducasse, L.; Galland, S.; Grelard, A.; Saucier, C.; Teissedre, P.L.; Dangles, O.; Quideau, S. Physicochemical Studies of New Anthocyano-Ellagitannin Hybrid Pigments: About the Origin of the Influence of Oak $C$-Glycosidic Ellagitannins on Wine Color. Eur. J. Org. Chem., 2010, (1), 55-63.

[66] Boulton, R. A Method for Assessment of Copigmentation in Red Wines Presented at the Forty-seventh Annual Meeting of the American Society of Enology and Viticulture, Reno NV, June 1996.

[67] Gomez-Miguez, M.; Gonzalez-Manzano, S.; Escribano-Bailon, M.T.; Heredia, F. J.; Santos-Buelga, C. Influence of Different Phenolic Copigments on the Color of Malvidin 3-Glucoside. J. Agric. Food Chem., 2006, 54(15), 5422-5429.

[68] Darias-Martin, J.; Carrillo-Lopez, M.; Echavarri-Granado, J.F.; DiazRomero, C. The Magnitude of Copigmentation in the Colour of Aged Red Wines Made in the Canary Islands. Eur. Food Res. Tech., 2007, 224(5), $643-$ 648.

[69] Levengood, J.; Boulton, R. The Variation in the Color Due to Copigmentation in Young Cabernet Sauvignon Wines. In Red Wine Color: Revealing the Mysteries. Waterhouse; Kennedy, Eds.; American Chemical Society: Washington DC, 2004; pp 34-52. 
[70] Schwarz, M.; Picazo-Bacete, J.J.; Winterhalter, P.; Hermosín-Gutiérrez, I. Effect of Copigments and Grape Cultivar on the Color of Red Wines Fermented After the Addition of Copigments. J. Agric. Food Chem., 2005, 53(21), 8372-8381.

[71] Hermosín-Gutiérrez, I.; Sanchez-Palomo, E.; Vicario-Espinosa, A. Phenolic Composition and Magnitude of Copigmentation in Young and Shortly Aged Red Wines Made from the Cultivars, Cabernet Sauvignon, Cencibel, and Syrah. Food Chem., 2005, 92(2), 269-283.

[72] Lorenzo, C.; Pardo, F.; Zalacain, A.; Alonso, G.L.; Salinas M.R. Effect of Red Grape Co-Winemaking in Polyphenols and Color of Wines. J. Agric. Food Chem., 2005, 53(19), 7609-7616.

[73] Versari, A.; Boulton, R.B.; Parpinello, G.P. A Comparison of Analytical Methods for Measuring the Color Components of Red Wines. Food Chemistry, 2008, 106(1), 397-402.

[74] Gonnet, J. F. Colour Effects of Co-Pigmentation of Anthocyanins Revisited 1. A Colorimetric Definition Using the CIELAB Scale. Food Chem., 1998, 63(3), 409-415.

[75] Gonnet, J. F. Colour Effects of co-pigmentation of anthocyanins revisited - 2. A colorimetric look at the solutions of cyanin co-pigmented by rutin using the CIELAB scale. Food Chem., 1999, 66(3), 387-394.

[76] Gordillo, B.; López-Infante, M.I.; Ramírez-Pérez, P.; González-Miret, M.L.; Heredia, F.J. Influence of Prefermentative Cold Maceration on the Color and Anthocyanic Copigmentation of Organic Tempranillo Wines Elaborated in a Warm Climate. J. Agric. Food Chem., 2010, 58(11), 6797-6803.

[77] Negueruela, A.I.; Echávarri, J.F.; Ayala, F.; Lomas, A.M. Colorimetría en Vinos. Zubia monográfico, 1995, 7, 151-166.

[78] Martinez, J.A.; Melgosa, M.; Perez, M.M.; Hita, E.; Negueruela, A.I. Visual and Instrumental Color Evaluation in Red Wines. Food Sci. Technol. Int., 2001, 7(5), 439-444.

[79] Mistry, T.V.; Cai, Y.; Lilley, T.H.; Haslam, E. Polyphenol Interactions. Part 5. Anthocyanin Copigmentation. J. Chem. Soc. Perkin Trans. 2, 1991, 1287-1296.

[80] Lewis, C.E.; Walker, J.R.L.; Lancaster, J.E. Effect of Polysaccharides on the colour of Anthocyanins. Food Chem., 1995, 54(3), 315-319.

[81] Guadalupe, Z.; Palacios, A.; Ayestaran, B. Maceration Enzymes and Mannoproteins: a Possible Strategy to Increase Colloidal Stability and Color Extraction in Red Wines. J. Agric. Food Chem., 2007, 55(12), 4854-4862.

[82] Eiro, M.J.; Heinonen, M. Anthocyanin Color Behavior and Stability During Storage: Effect on Intermolecular Copigmentation. J. Agric. Food Chem., 2002, 50(25), 7461-7466.

[83] Fernandez de Simon, B.; Hernandez, T.; Cadahia, E.; Dueñas, M.; Estrella, I. Phenolic Compounds in a Spanish Red Wine Aged in Barrels Made of Spanish, French and American Oak Wood. Eur. Food Res. Tech., 2003, 216(2), 150-156.

[84] Perez-Prieto, L.J.; de la Hera-Orts, M.L.; Lopez-Roca, J.M.; FernandezFernandez, J.I.; Gomez-Plaza, E. Oak-Matured Wines: Influence of the Characteristics of the Barrel on Wine Colour and Sensory Characteristics. $J$. Sci. Food Agric., 2003, 83(14), 1445-1450.

[85] Davies, A.J.; Mazza, G. Copigmentation of Simple and Acylated Anthocyanins with Colorless Phenolic Compounds. J. Agric. Food Chem., 1993, 41(5), 716-720.

[86] Baranac, J.M.; Petranovic, N.A.; Dimitric-Markovic, J.M. Spectrophotometric Study of Anthocyan Copigmentation Reactions. 2. Malvin and the Nonglycosidized Flavone Quercetin. J. Agric. Food Chem., 1997, 45(5), 1694-1697.

[87] Baranac, J.M.; Petranovic, N.A.; Dimitric-Marcovic, J.M. Spectrophotometric Study of Anthocyanin Copigmentation Reactions. 2. Malvin and the Nonglycosidized Flavone Morin. J. Agric. Food Chem., 1997, 45(5), 16981700 .

[88] Cheynier, V.; Moutounet, M.; Sarni-Manchado, P. Los Compuestos Fenólicos. In Enologia: Fundamentos Cientificos y Tecnologicos; Flanzy, Ed.; Mundi-Prensa: Madrid, 2003; pp. 114-136.
[89] Hollman, P.C.H.; Arts, I.C.W. Flavonols, Flavones and Flavanols - Nature, Occurrence and Dietary Burden. J. Sci. Food Agric., 2000, 80(7), pp 10811093.

[90] McDonald, M.S.; Hughes, M.; Burns, J.; Lean, M.E.J.; Matthews, D.; Crozier, A. Survey of the Free and Conjugated Myricetin and Quercetin Content of Red Wines of Different Geographical Origins. J. Agric. Food Chem., 1998, 46(2), 368-375.

[91] Clifford, M.N. Anthocyanins - Nature, Occurrence and Dietary Burden. $J$. Sci. Food Agric., 2000, 80(7), 1063-1072.

[92] Santos-Buelga, C.; Scalbert, A. Proanthocyanidins and Tannin-Like Compounds - Nature, Occurrence, Dietary Intake and Effects on Nutrition and Health. J. Sci. Food Agric., 2000, 80(7), 1094-1117.

[93] Liao, H.; Cai, Y.; Haslam, E. Polyphenol Interactions. Anthocyanins: CoPigmentation and Colour Changes in Red Wines. J. Sci. Food Agric., 1992 59(3), 299-305.

[94] Mirabel, M.; Saucier, C.; Guerra, C.; Glories, Y. Copigmentation in Model Wine Solutions: Occurrence and Relation to Wine Aging. Am. J. Enol. Vitic., 1999, 50(2), 211-218.

[95] Berké, B.; De Freitas, V.A.P. A Colorimetric Study of Oenin Copigmented by Procyanidins. J. Sci. Food Agric., 2007, 87(2), 260-265.

[96] De Freitas, V.A.P.; Glories, Y.; Bourgeois, G.; Vitry, C. Characterisation of Oligomeric and Polymeric Procyanidins from Grape Seeds by Liquid Secondary Ion Mass Spectrometry. Phytochemistry, 1998, 49(5), 1435-1441.

[97] Berké, B.; De Freitas, V.A.P. Influence of Procyanidin Structures on Their Ability to Complex with Oenin. Food Chem., 2005, 90 (3), pp 453-460.

[98] Escribano-Bailon, M.T.; Santos-Buelga, C.; Francia-Aricha, E.M.; RivasGonzalo, J.C.; Heredia, F.J. Flavanol-Anthocyanin Copigmentation and Colour Quality. In Proceedings of 1st International Congress on Pigments in Food Technology. Sevilla, Spain, 1999; pp. 363-367.

[99] Malien-Aubert, C.; Dangles, O.; Amiot, M.J. Influence of Procyanidins on the Color Stability of Oenin Solutions. J. Agric. Food Chem., 2002, 50(11), 3299-3305.

[100] Gonzalez-Manzano, S.; Mateus, N.; De Freitas, V.A.P.; Santos-Buelga, C. Influence of the Degree of Polymerisation in the Ability of Catechins to Act as Anthocyanin Copigments. Eur. Food Res. Tech., 2008, 227(1), 83-92.

[101] Darias-Martín, J.; Carrillo, M., Díaz, E.; Boulton R.B. Enhancement of Red Wine Colour by Pre-Fermentation Addition of Copigments. Food Chem., 2001, 73(2), 217-220.

[102] Darias-Martín, J.; Martin-Luis, B.; Carrillo-Lopez, M.; Lamuela-Raventos, R.; Díaz-Romero, C.; Boulton R.B. The Effect of Caffeic Acid on the Color of Red Wine. J. Agric. Food Chem., 2002, 50(7), 2062-2067.

[103] Bloomfield, D.G.; Heatherbell, D.A.; Nikfardjam, M.S.P. Effect of $p$ Coumaric Acid on the Color in Red Wine. Mitt. Klosterneuburg, 2003, 53(56), 195-198

[104] Kovac, V.; Alonso, E.; Bourzeix, M.; Revilla, E. Effect of Several Enological Practices on the Content of Catechins and Proanthocyanidins of Red Wines. J. Agric. Food Chem., 1992, 40(10), 1953-1957.

[105] Kovac, V.; Alonso, E.; Revilla, E. The Effect of Adding Supplementary Quantities of Seeds During Fermentation on the Phenolic Composition of Wines. Am. J. Enol. Vitic., 1995, 46(3), 363-367.

[106] Canals, R.; Llaudy, M.C.; Canals, J.M.; Zamora, F. Influence of the Elimination and Addition of Seeds on the Colour, Phenolic Composition and Astringency of Red Wine. Eur. Food Res. Tech. 2008, 226(4), 1183-1190.

[107] Markovic, J.M.D.; Ignjatovic, L.M; Markovic, D.A; Baranac, J.M. Antioxidative Capabilities of Some Organic Acids and Their Co-Pigments with Malvin Part I. J. Electroanal. Chem., 2003, 553, 169-175.

[108] Alvarez, I.; Aleixandre, J.L.; Garcia, M.J.; Lizama, V.; Aleixandre-Tudo, J.L. Effect of the Prefermentative Addition of Copigments on the Polyphenolic Composition of Tempranillo Wines After Malolactic Fermentation. Eur Food Res. Technol., 2009, 228(4), 501-510. 